\title{
ESTRUTURA E DINÂMICA FLORESTAL, ANTES E APÓS EXTRAÇÃO DE MADEIRA, EM ÁREA DE MANEJO FLORESTAL NA FLONA DO TAPAJÓS
}

\author{
Maiki de Matos Natividade'; Juliane da Silva Sampaio; Waldeir dos Santos Pereira3; lara Rayana Leal \\ de Sousa ; Cezar Dias Cardoso Júnior ${ }^{5}$; Cezarina do Socorro de Souza Carvalho ${ }^{6}$; Lia de Oliveira \\ Melo ${ }^{7}$

\footnotetext{
1 Universidade Federal do Oeste do Pará, Santarém, Pará, Brasil, natividade.legal@gmail.com

2 Universidade Federal do Oeste do Pará, Santarém, Pará, Brasil, julianesampaio22@gmail.com;

3 Universidade Federal do Oeste do Pará, Santarém, Pará, Brasil, waldeir.santtos@hotmail.com

${ }^{4}$ Universidade Federal do Oeste do Pará, Santarém, Pará, Brasil, rayana.lealgirl@hotmail.com

${ }^{5}$ Universidade Federal do Oeste do Pará, Santarém, Pará, Brasil, cezar.cardosojr@gmail.com

6 Universidade Federal do Oeste do Pará, Santarém, Pará, Brasil, cezarina13@gmail.com

7 Universidade Federal do Oeste do Pará, Santarém, Pará, Brasil, Icolivei@gmail.com
}

RESUMO: A dinâmica florestal tem finalidade de analisar o comportamento de uma floresta em relação a sua estrutura, composição, crescimento, mortalidade, regeneração e fatores ecológicos diante de modificações causadas por efeitos naturais ou antrópicos. Neste sentido o objetivo deste trabalho foi avaliar os efeitos da extração de madeira de impacto reduzido sobre a estrutura e dinâmica florestal em uma área de manejo florestal comunitário com 1.600 ha localizada na Floresta Nacional do Tapajós. Os dados coletados são oriundos de 08 parcelas permanentes de monitoramento florestal com 0,25 ha cada mensuradas antes e após a colheita de madeira. Foram observados os seguintes parâmetros: estrutura da floresta, índice de valor de importância, distribuição diamétrica pré e pós-exploração e dinâmica florestal. Os dados coletados em campo foram inseridos e analisados no programa Monitoramento de Florestas Tropicais (MFT). A extração de madeira realizada na área não afetou a estrutura da floresta, pois a vegetação manteve a distribuição de classe diamétrica, alta produtividade com baixo impacto, reafirmando assim que o plano de manejo realizado na área é eficiente.

PALAVRAS-CHAVE: Monitoramento, Mortalidade, Impacto reduzido.

\section{FOREST STRUCTURE AND DYNAMICS, BEFORE AND AFTER THE EXTRACTION OF WOOD, IN A FOREST MANAGEMENT AREA OF THE FLONA TAPAJOS}

ABSTRACT: Forest dynamics has the purpose of analyzing the behavior of a forest in relation to its structure, composition, growth, mortality, regeneration and ecological factors in face of modifications caused by natural or anthropic effects. In this sense, the objective of this work was to evaluate the effects of reduced impact logging on forest structure and dynamics in a 1600ha community forest management area located 
in the Tapajós National Forest, Km 117. The data collected come from 08 plots forest monitoring with 0.25 ha each measured before and after harvesting. The following parameters were observed: forest structure, importance value index, pre and postharvest diametric distribution and forest dynamics. The data collected in the field were inserted and analyzed in the Tropical Forest Monitoring (MFT) program. The extraction of wood made in the area did not affect the structure of the forest, as it maintained the diametric class distribution, high productivity with low impact, thus reaffirming that the management plan carried out in the area is efficient.

KEYWORDS: Monitoring, Mortality, Reduced impact.

\section{ESTRUCTURA Y DINÁMICA FORESTAL, ANTES Y DESPUÉS DE LA EXTRACCIÓN DE MADERA, EN ÁREA DE MANEJO FORESTAL EN FLONA DO TAPAJÓS}

RESUMEN: La dinâmica forestal tiene el propósito de analizar el comportamiento de un bosque em relación a su estructura, composición, crecimiento, mortalidad, regeneración y factores ecológicos ante modificaciones causadas por efectos naturales o antrópicos. E neste sentido el objetivo de este trabajo fue evaluar los efectos de la extracción de madera de impacto reducido sobre la estructura y dinâmica forestalen un área de manejo forestal comunitario con 1600ha ubicadaen el Bosque Nacional del Tapajós, Km 117. Los datosre colecta dos proceden de 08 parcelas permanentes de monitoreo forestal con 0,25 ha cada mensuradas antes y después de la cosecha de madera. Se observa ronlos siguientes parámetros: estructura del bosque, índice de valor de importancia, distribución diamétricapre y post-exploración y dinâmica forestal. Los datos recolectado sen campo fueroninsertados y analizadosen el programa Monitoreo de Bosques Tropicales (MFT). La extracción de madera realizada en el área no afectó la estructura del bosque, puesmantuvo la distribución de clase diamétrica, alta productividad con bajo impacto, reafirmando así que el plan de manejo realizado en el área es eficiente.

PALABRAS-CLAVE: Impacto reducido, Monitoreo, Mortalidad.

\section{INTRODUÇÃO}

A biodiversidade do planeta tem grande contribuição das formações florestais, tornando explicita o alto valor da floresta Amazônica. Diante disso, seus recursos apresentam elevado potencial de matérias-primas e de bens e serviços para a sociedade, 
que são explorados de forma tradicional, além da prática de colheita no manejo florestal. A estimativa do crescimento é essencial para o plano de manejo sustentável. Conhecer o crescimento de uma árvore ou floresta é de grande interesse no meio florestal, sendo possível usar essas informações para definir a rotação silvicultural e prognosticar a produção florestal (VANCLAY, 1994).

Para avaliar o caráter dinâmico de uma floresta após uma intervenção exploratória dos recursos madeireiros, faz-se necessário proceder ao monitoramento (QUEIROZ, 1998; PÉLLICO NETTO; BRENA, 1997). Esse procedimento é conhecido como Inventário Florestal Contínuo (IFC) e corresponde a efetuar medições na floresta repetidas vezes no tempo. Tal procedimento resulta na obtenção de uma série de informações fundamentais aos manejadores, como crescimento, ingresso, mortalidade, ciclo de corte, sucessão e densidade de estoque, entre outros.
A dinâmica florestal, tem finalidade de analisar o comportamento de uma floresta em relação a sua estrutura, composição, crescimento, mortalidade, regeneração, fatores ecológicos, diante modificações causadas por efeitos naturais ou antrópicos, nos quais podem afetar tomada de decisão na colheita (SOUZA et al., 2012).

A composição florística é um fator essencial para se desenvolver estudos adicionais sobre a estrutura e a dinâmica da floresta (CARVALHO,1997). A relação deste fator com a distribuição em classes de diâmetro dos indivíduos estudados permite um conhecimento mais consolidado da floresta estudada, como afirma Finger (2006), quando coloca que a distribuição diamétrica é caracterizada de acordo com informações em intervalos de classe, permitindo uma melhor visualização da estrutura da floresta em relação ao seu crescimento, por isso Ferreira et al (1998) afirmam que é importante obter esse estudo para conhecer o estoque da floresta. 
Neste sentido o objetivo deste trabalho foi avaliar os efeitos da extração de madeira, com impacto reduzido, sobre a estrutura da floresta e as taxas de crescimento, ingressos e mortalidade de árvores em uma área manejada na Floresta Nacional do Tapajós.

\section{MATERIAL E MÉTODOS}

O presente estudo foi realizado em uma área de manejo florestal comunitário na Floresta Nacional do Tapajós (FLONA do Tapajós), localizada no KM 117 da BR 163, município de Belterra, Pará. O clima da região, pela classificação de Koppen, é do tipo Ami, com temperatura média anual de $25,5^{\circ} \mathrm{C}$. A concentração de chuvas ocorre entre janeiro e maio, resultando em uma precipitação média anual de 1.820 mm (IBAMA, 2004). A Flona Tapajós abrange o bioma Amazônia e a tipologia florestal predominante é Floresta Ombrófila Densa de terra firme.

Os dados coletados são oriundos de parcelas permanentes monitoramento florestal que estão inseridas em uma unidade de produção anual de madeira (UPA 11), que possui 1.600 ha. Foram medidas 8 parcelas de $50 \mathrm{~m} \times 50 \mathrm{~m}$, distribuídas aleatoriamente na área. Cada parcela foi subdividida em 25 subparcelas de 10 m x10 m. A primeira medição ocorreu no ano de 2016, período que antecedeu a exploração madeireira. Em julho de 2017, após o período exploratório ocorreu a segunda medição destas parcelas.

Nas subparcelas, todas as árvores com CAP (circunferência à altura do peito), maior ou igual a $32,0 \mathrm{~cm}$, foram identificadas com uma plaqueta de alumínio contendo numeração própria e tiveram seu ponto de medição do CAP marcado com tinta à base de óleo na cor vermelha, a fim de diminuir a possibilidade de erros em medições posteriores. O CAP foi medido com fita métrica com precisão de milímetros. Na segunda medição foram registradas as árvores caídas, quebradas, danificadas e mortas a fim de verificar os ingressos e mortalidade de 
indivíduos, bem como a situação dos mesmos pós-exploração.

A medição seguiu as diretrizes publicadas em Silva et al 2005. A identificação das espécies foi feita com auxílio de profissionais que atuam como identificadores botânicos ligados a Cooperativa Mista da Floresta Nacional do Tapajós (COOMFLONA).

Os dados coletados foram inseridos e analisados no programa Monitoramento de Florestas Tropicais (MFT), criado pela Embrapa Amazônia Oriental.

\section{RESULTADOS E DISCUSSÃO}

A Unidade de Produção Anual (UPA 11), no ano de 2016, quando a floresta não havia sofrido intervenção, apresentou 382,5 indivíduos.ha-1 ${ }^{\text {, área }}$ basal de 28,115 $\mathrm{m}^{2}$.ha-1 e volume de $317,84 \mathrm{~m}^{3} \cdot \mathrm{ha}^{-1}$. Após a exploração, houve um decréscimo nesses valores sendo contabilizados 346,5 indivíduos.ha ${ }^{-1}$, área basal de 25,932 $\mathrm{m}^{2}$.ha $\mathrm{A}^{-1}$ e volume de 294,36 $\mathrm{m}^{3} \cdot \mathrm{ha}^{-1}$. A redução de 9,4\% no número de árvores, 7,8\% na área basal e 7,4\% do volume original da floresta foi, em sua maior parte, causada pelas atividades de extração de madeira (derruba e arraste das toras) além da mortalidade devido a causas naturais ocorrida no intervalo de tempo entre as duas medições.

No ano de 2016, período que antecedeu a exploração, foram contabilizadas um total de 153 espécies, dentre estas, as 10 espécies que apresentaram maior Índice de Valor de Importância (IVI) foram: Matamatá-preto (Eschweilera sp.), Acariquara (Minquartia guianensis), Abiurana (Pouteria sp.), Acariquarana (Rinorea guianensis), Pente-de-macaco (Apeiba macropetala), Quinarana (Geissospermum sericeum), Tauarí (Couratari guianensis), Maçaranduba (Manilkara sp.), Breu-amarelo (Protium paniculatum) e Muiratinga (Maquira sp.) (Figuras 1). Após a exploração, ano de 2017, foram contabilizadas 150 espécies, mantendo praticamente as mesmas espécies no ranking do IVI, porém, Acariquarana e Abiurana inverteram suas posições. Essa 
mudança de posição no ranking, estava entre as 10 espécies de maior IVI explica-se pela perda de indivíduos no ano de 2016, saiu do ranking no ano retirados da floresta durante a extração de 2017, sendo substituída pelo Breude madeira. O Breu amarelo, que vermelho (Protium sp.) (Figura 1).

Figuras 1. Espécies com maior Índice de Valor de Importância nos anos de 2016 (A) e 2017 (B), período pré e pós-exploração.
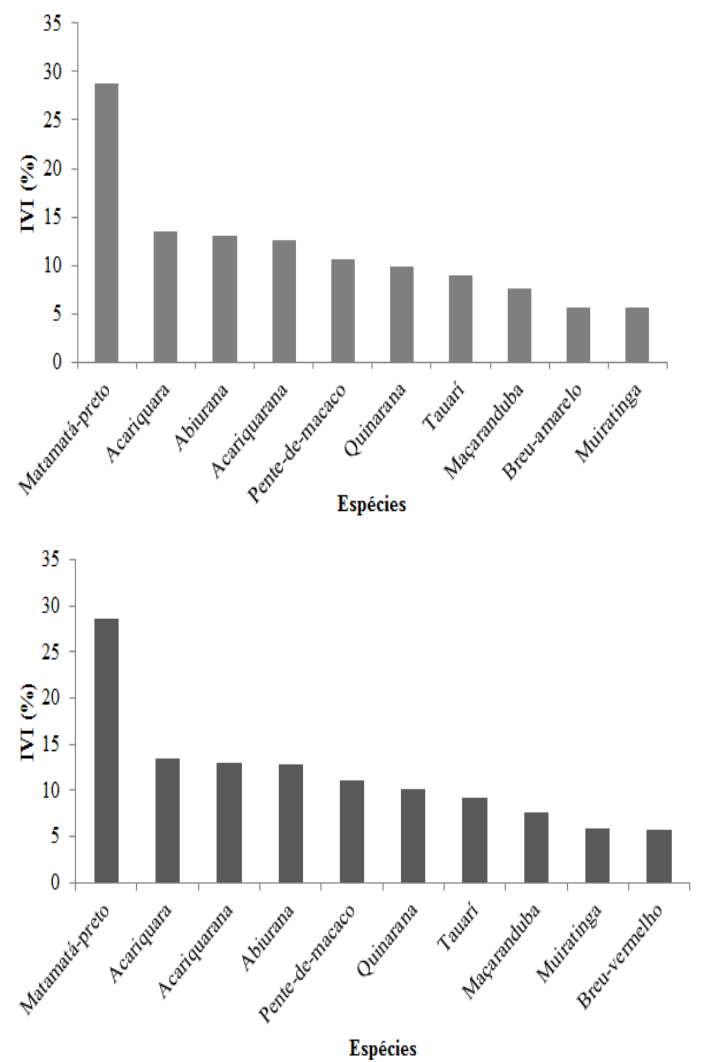

Dentre as espécies identificadas na área de manejo mais de $60 \%$ são de interesse comercial na região e utilizadas para diversas finalidades. Gonçalves \& Santos (2008), identificaram a mesma realidade ao analisarem outra área de manejo florestal que foi instalada na porção norte dentro da Floresta Nacional do Tapajós.

As florestas nativas, geralmente, caracterizam-se por apresentar 
distribuição diamétrica decrescente, em forma de 'J-invertido'. Segundo Lima \& Leão (2013) essa característica é encontrada quando há maior quantidade de indivíduos nas classes de tamanhos menores, diminuindo gradativamente com o aumento das classes. Na UPA 11, observou-se que a estrutura da floresta estudada, em 'J- invertido', não sofreu alterações abruptas que afetassem a distribuição, mesmo após o período de exploração (Figura 2 A e 2 B). Gomes et al. (2004), observou as mesmas características ao analisar uma área de floresta manejada, antes e após seu período de exploração no estado da Paraíba.

Figura 2. Distribuição em classes de diâmetro nos anos de 2016 (A) e 2017(B), nos períodos de pré e pós-exploração.

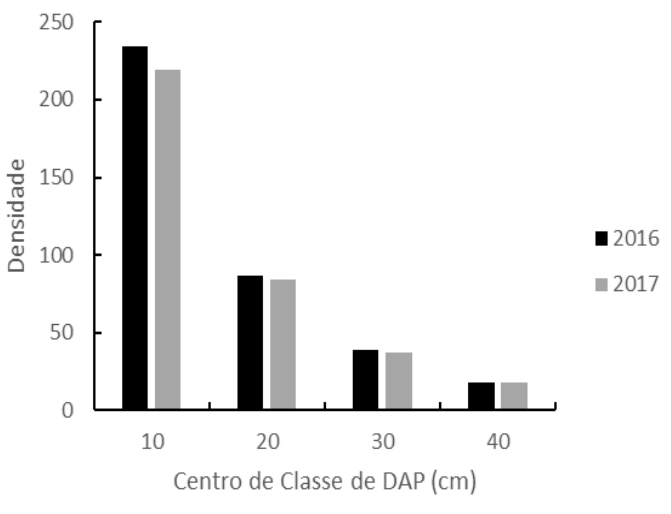

As principais classes diamétricas que sofreram redução após a colheita florestal foram as $10-19,9 \mathrm{~cm}, 50-59,9$ $\mathrm{cm}, 60-69,9 \mathrm{~cm}$ e $>110 \mathrm{~cm}$ que tiveram sua densidade diminuída em 6,20\%, $7,41 \%, \quad 23,08 \%$ e $33,33 \%$, respectivamente. A redução da

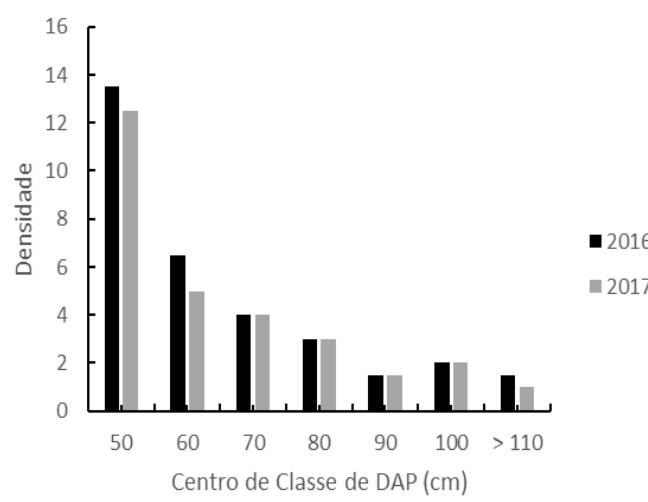

densidade nas classes de DAP a partir de $50 \mathrm{~cm}$, deve-se principalmente a colheita florestal cujo diâmetro mínimo de corte no manejo foi de $55 \mathrm{~cm}$ (Figura 2 B).

O incremento médio anual em diâmetro considerando todas as 399 
árvores monitoradas foi de 0,44 $\mathrm{cm}^{\mathrm{anno}}{ }^{-1}$, as 179 que recebiam luz

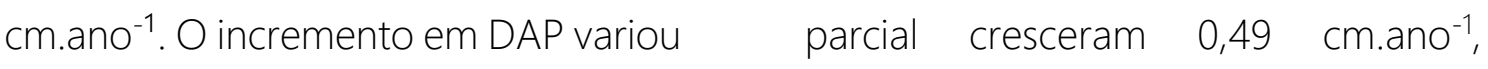
conforme o nível de iluminação enquanto que as 168 árvores recebida pela copa das árvores (Figura completamente sombreadas (luz 5). As 52 árvores que apresentavam difusa) obtiveram crescimento de 0,31 copas completamente expostas à luz $\quad \mathrm{cm}^{\mathrm{anno}}{ }^{-1}$. obtiveram incremento anual de 0,66

Figura 3. Incremento médio anual relacionado com o grau de iluminação de copa, na área da Floresta Nacional do Tapajós, Belterra-PA.

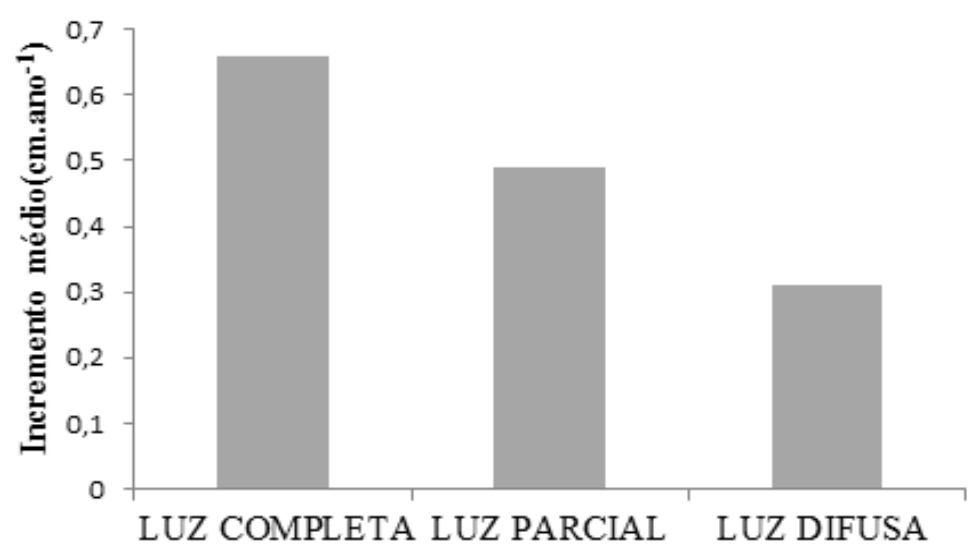

O valor do incremento médio em diâmetro obteve um decréscimo em relação ao grau de iluminação das copas das árvores. As árvores que estavam presentes no dossel da floresta receberam maior quantidade de luz, logo obtiveram maiores valores de incremento médio anual quando comparadas com as árvores que receberam luz parcial, que tiveram um leve decréscimo no seu crescimento. As árvores que obtiveram menores valores médios de incremento foram aquelas que estavam presentes no sub-bosque e que receberam apenas iluminação difusa. A quantidade de luz recebida é um fator preponderante para o crescimento em diâmetro e 
altura da maioria das espécies. Silva et al. (1995) afirmam existir forte correlação entre o grau de iluminação da copa e o crescimento das árvores. Segundo Walter (1971) a distribuição local das espécies em uma comunidade florestal está fortemente influenciada pelas diferenças na disponibilidade de luz, que condiciona direta ou indiretamente grande parte dos processos de crescimento das plantas. A intensidade de iluminação, também está ligada ao nível de perturbação da floresta, mais especificamente do seu dossel (VATRAZ et al., 2015).

As taxas de mortalidade, sobrevivência e ingressos na UPA 11, no ano de 2017 estão presentes na tabela 1. O número de indivíduos ingressos no ano de 2017 foi de 10,67 ha. $^{-1}$.ano ${ }^{-1}$ (1,96\%). A mortalidade, representada pelos indivíduos medidos em 2016 que não estavam mais presentes no ano de 2017, devido a exploração ou por morte natural, foi de 39,33 ha. ${ }^{-1}$. ano $^{-1}$ correspondendo a 7,21\% do número total de indivíduos medidos.

Tabela 1. Taxa de mortalidade, ingressos e sobreviventes na UPA 11 referentes a área da Floresta Nacional do Tapajós, Belterra-PA.

\begin{tabular}{c|c|c|c|c|c}
\hline \multicolumn{2}{c|}{ Mortalidade } & \multicolumn{2}{c|}{ Ingressos } & \multicolumn{2}{c}{ Sobrevivência } \\
\hline M/ha/ano & $\%$ & I/ha/ano & $\%$ & S/ha & $\%$ \\
\hline 39,33 & 7,21 & 10,67 & 1,96 & 379,5 & 92,79 \\
\hline
\end{tabular}

○ povoamento demonstrou extração de madeira, porém com o balanço negativo com a taxa de avançar do tempo esta tendência mortalidade sendo superior à de costuma se inverter, pois as clareiras ingressos de novos indivíduos, abertas durante a colheita estimulam a consequência natural de áreas recém regeneração natural e o crescimento manejadas, onde a mortalidade é das árvores remanescentes, elevando maior nos primeiros anos após a assim o número de ingressos. Souza et 
al. 2017 verificou o mesmo balanço entre taxa de mortalidade e ingressos em um estudo realizado em uma área de Manejo Florestal Sustentável, localizada entre os municípios de Itacoatiara, Silves e Itapiranga, no Amazonas.

A taxa de sobrevivência de indivíduos dos anos de 2016 para 2017 foi de 92,79\%, valor que pode ser considerado alto, inferindo-se que a extração de madeira que aconteceu na área, não causou danos severos aos indivíduos remanescentes.

\section{CONCLUSÃO}

A exploração florestal realizada na UPA 11, reduziu o plantel de árvores do povoamento, porém não provocou alterações na estrutura da floresta, que manteve sua distribuição diamétrica e fitossociologia.

A exposição dos indivíduos a luz completa resultou em maior incremento médio em relação aos indivíduos localizados em luz difusa, evidenciando a importância da exposição solar no crescimento arbóreo.

\section{REFERÊNCIAS}

CARVALHO, J. O. P. Dinâmica de florestas naturais e sua implicação para o manejo florestal (Curso de manejo florestal sustentável). Curitiba:

EMBRAPA Florestas, 1997.

FERREIRA, R. L. C.; SOUZA, A. L.; JESUS, R.M. de. Dinâmica da estrutura de uma floresta secundária de transição. Distribuição diamétrica. Revista árvore, Viçosa, v. 22, n.3, p. 331-344,1998.

FINGER, C. A. G. Biometria florestal. Santa Maria. Universidade Federal de Santa Maria, 2006.284p.

FRANCEZ, L. M. B.; CARVALHO, J. O. P.; BATISTA, F. J.; JARDIM, F. C. S.; RAMOS, M. L. S. Influência da exploração florestal de impacto reduzido sobre as fases de desenvolvimento de uma floresta de terra firme, Pará, Brasil. Ciência Florestal, Santa Maria, v. 23, n. 4, p. 743-753, 2013.

GOMES, A. P. C.; SOUZA, A. L. de; MEIRA NETO, J. A. A. Alteração estrutural de uma área florestal explorada convencionalmente na bacia do Paraíba do Sul, Minas Gerais, nos domínios de Floresta Atlântica. Revista Árvore, v.28, n.3, p. 407-417, 2004.

GONÇALVES, Fábio Guimarães; SANTOS, JR dos. Composição florística e estrutura de uma unidade de manejo florestal sustentável na Floresta Nacional do Tapajós, Pará. Acta Amazonica, v. 38, n. 2, p. 229-244, 2008. 
INSTITUTO BRASILEIRO DO MEIO AMBIENTE E DOS RECURSOS NATURAIS RENOVÁVEIS - IBAMA. Floresta Nacional do Tapajós: Plano de Manejo. Belterra, 2004.

JARDIM, F. C. S.; SERRÃO, D.R.; NEMER, T.C. Efeito de diferentes tamanhos de clareiras, sobre o crescimento e a mortalidade de espécies arbóreas, em Moju-PA. Acta Amazonica, v.37, n.1, p.37-48, 2007.

LIMA, J. P. da C.; LEÃO, J. R. A. Dinâmica de crescimento e distribuição diamétrica de fragmentos de florestas nativa e plantada na Amazônia sul ocidental. Floresta e Ambiente, Edição: jan./mar., p. 70-79, 2013.

PÉllico NETTO, S. BRENA, D. A. Inventário Florestal. Curitiba: Editado pelos autores. 316 p.1997. QUEIROZ, W. T. Técnicas de amostragem em inventário florestal nos Trópicos. Belém: FCAP. Serviço de Documentação e Informação. 1998. 147 p.

SILVA, J. N. M.; CARVALHO, J. O. P.; LOPES, J. DO C. A.; ALMEIDA, B. F. DE; COSTA, D. H. M.; OLIVEIRA, L. C. DE; VANCLAY, J. K.; SKOVSGAARD, J. P. Growthand yield of a tropical rain forest in the Brazilian Amazon 13 yearsaf terlogging. Forest Ecologyand Management. n.71, p.267-274, 1995.

SILVA, J. N. M.; LOPES, J. C. A.; OLIVEIRA, L. C.; SILVA, S. M. A.; CARVALHO, J. O. P.; COSTA, D. H. M.; MELO, M. S.; TAVARES, M. J. M. Diretrizes para a instalação e medição de parcelas permanentes em florestas naturais da Amazônia Brasileira. EMBRAPA Amazônia Oriental, Belém, Pará. 2005.

SILVA, M. R.; ARAÚJO, G.M. Dinâmica da comunidade arbórea de uma floresta semidecidual em Uberlândia, MG, Brasil. Acta Bot. Bras., v.23, n.1, p.49-56, 2009.

SOUZA, C. R.; AZEVEDO, C.P.; ROSSI, L.M.B.; SILVA, K.E.; SANTOS, J.; HIGUCHI, N. Dinâmica e estoque de carbono em floresta primária na região de Manaus/AM. Acta Amazonica, v.42, n.4, p.501-506, 2012.

SOUZA, M. A. S.; AZEVEDO, C. P.; SOUZA, C. R. FRANÇA, M.; NETO, E. L. $\mathrm{V}$.

Dinâmica e produção de uma floresta sob regime de manejo sustentável na Amazônia central. Floresta, v. 47, n. 1, p. 55 - 63, jan. / mar. Curitiba, 2017.

VATRAZ, S.; CARVALHO, J. O. P.; SILVA, J. N. M.; CASTRO, T.C. Efeito da exploração de impacto reduzido na dinâmica do crescimento de uma floresta natural. Scientia Forestalis, Piracicaba, v. 44, n. 109, p. 261-271, 2015.

VANCLAY, J. K. Modeling forest grow thand yield: applications to mixed tropical forests. Copenhagen: $C A B$ International, 1994. 312 p

WALTER, H. Ecologyof tropical and subtropical vegetation. Edinburg: Oliver \& Boyd, 1971, p. 1-29. 